\title{
An Advanced Methodology of Visibility Used for Underwater Image Enhancement
}

D.O.I-10.51201/12452

\section{https://doi.org/10.51201/12452}

\author{
Smita Nagtode \\ Department of Electronics \& Telecommunication engineering, DMITER, Sawangi (M). \\ Arati Kadve \\ Department of Electronics \& Telecommunication engineering, DMITER, Sawangi (M). \\ Dinesh Bhoyar
}

Department of Electronics \& Telecommunication engineering, YCCE, Nagpur

\begin{abstract}
This paper concentrates towards submerged processing of image so as to improve the picture quality. As the greater part of the pictures of seaward establishments, drinking water store and so forth are caught and examined physically by divers. And manual intervention in this regard is dangerous, costly, and time-consuming and yet does not often enable a full assessment. Hence camera based inspection is used to capture the images under water. Using cameras underwater poses major technological challenges. The objects in the submerged images are pale, difficult to sight and examine because the images of such environment loses the details of the object. The articles in the submerged pictures are swoon, hard to see and examine in light of the fact that the pictures of such condition loses the subtleties of the item. The submerged pictures typically experience the ill effects of non-uniform lighting, low difference, slant, obscures and lessened hues. Also, consequently in this examination work, a novel technique has been proposed for dealing with submerged picture slanting and obscuring if there should be an occurrence of unidirectional cyclic waves and roundabout waves to upgrade the perceivability of submerged pictures. The proposed work develops a geometrical model for image restoration from these distortions with good accuracy.
\end{abstract}

Index Terms - Underwater image correction, visibility restoration, image dehazing, visibility enhancement.

\section{INTRODUCTION}

There is an ample research field in the subaquatic image processing area as the objective of exploring and investigating subaquatic image activities. However, the underwater images captured lack quality and visibility. Therefore, it is necessary to improve the quality of the underwater images and to improve their visibility. The underwater image processing finds its application in areas such as plant inspection, seabed exploration and search of shipwrecks for exploration of natural resources. Due to more visibility conditions, or environment two oceans of the world have not yet been explored. And many techniques for the improvement of underwater images are available here, since Terra is an aquatic planet that has $70 \%$ of its surface covered by water. Scientists show their great interest in knowing what is under water, and also, this field has given importance to the use of subaquatic sequences to monitor marine species and subaquatic plants, to achieve this purpose and absolutely necessary use of clear and qualitative underwater images. 
Problems associated with subaquatic images: -Many problems arise, such as diffusion and crinkling of pattern due to reflection gives light. In reflected light it is partially polarized horizontally and partially enters the water vertically. Due to vertical polarization, or underwater object that is less bright and observes deep cores.

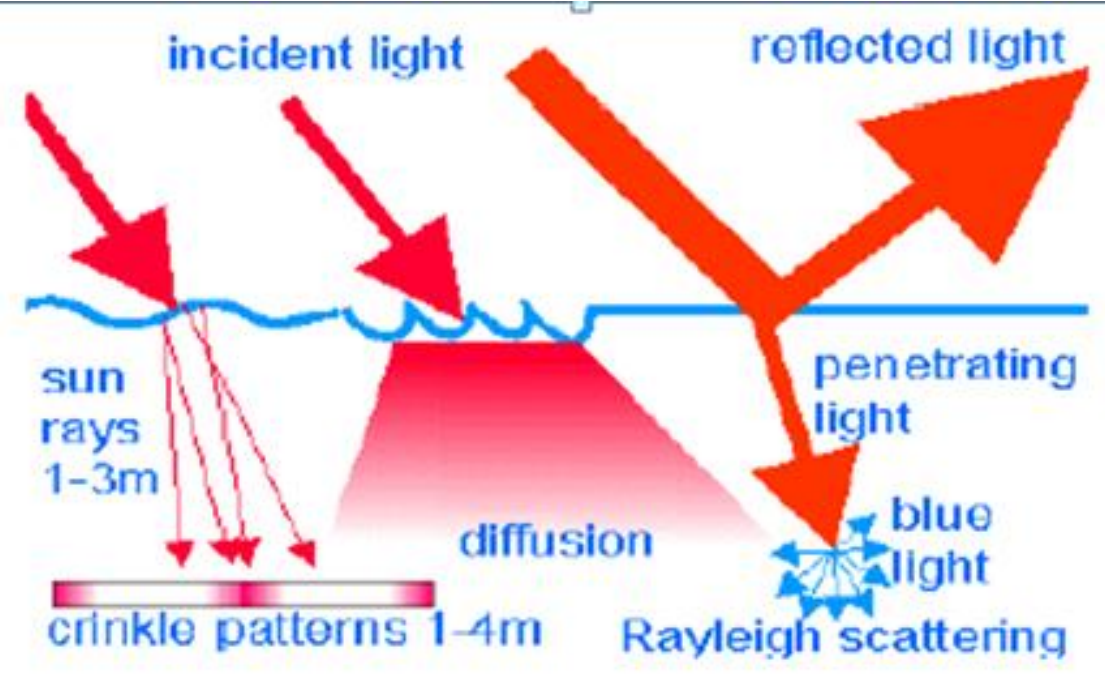

Figure 1. Effect of light on water surface

\begin{abstract}
Also, there is another problem related to water density, which is denser than it is. Therefore, when the rays of light move to the water, they are partially reflected reverse and, at the same time, partially entered the water. The amount of light is reduced as it goes to or from the sea. Thus water molecules also absorb a certain amount of light. As a result, subaquatic images darken as depth increases. Not just a quantity of light rays is condensed when you are more founded, but also cores fall to a number, depending on the wave compression of the cores. For example, first, all red disappears to a depth of $3 \mathrm{~m}$. In second place, orange colour is beginning to disappear as we advance. At a depth of $5 \mathrm{~m}$, on the edge and lost. There are several methods for processing underwater images. In general, the methods are classified according to the approaches: methods based on hardware and methods based on software. Here four traditional approaches of hardware for obtaining sub-aquatic images: polarization, images controlled by rank, fluorescence images and stereo images Light has the properties of intensity, wavelength and polarization. Natural light does not have polarization, but the light that uses an image sensor has little polarization.
\end{abstract}

\title{
II. LITERATURE REVIEW
}

In addition to adopting the blurry image with the image formation model (IFM) [2] there is a way to estimate the distance between the points of the scene and the camera and, therefore, recover and enhance the underwater images. This document uses the blurred image to estimate the depth map to improve the underwater image. It is based on the observation that the poorer objects of the camera are more blurry for underwater images.

The contrast and low contrast with the characteristics of underwater images, which are similar to nebulous images, is the main challenge in the search for pieces from underwater images. To overcome the effects of blurring and low contrast, apply the darkness of the channel first [3], which aims to eliminate the fog from a single input image. Since underwater images are similar to fog images, this method can also be applied to underwater images. The previous dark channel is based on the most local patches on exterior images without fog that contain some pixels, that have lower intensities at least one colour channel. Using the previous one with the fog image model, you can retrieve a high quality fog image. When the dark channel is applied to a submarine image, a clear image is generated. 
The objects are very different in the underwater environment compared to their appearance in sunlight. High quality images with correct colours simplify the detection of underwater objects and can allow the use of visual SLAM algorithms developed for terrestrial robots under water. Therefore, the processing of images is required to obtain high quality images and correct colouring. The current algorithms are entered in the reconstruction of the colour [4] of the landscape in the depth of the block, which means sunlight is still present and can also distinguish different colours. To a greater depth, the filtrate is much stronger, so it is not possible. This studio investigates whether it automatically learns whether it can be used to transform image data. In order to obtain images and sub-aquatic lighting conditions in a controlled environment, a special light source with a defined wavelength is used for the illumination of objects in a laboratory setting. The images are fed through statistical learning algorithms with no previous filters. It shows that there are machines in poor surroundings and support vectors that are bad for the given field and produce excellent results.

The filtration methods in the spatial domain and the frequency and the linear filtrate are conceptually pleasant and extremely useful in many applications [6]. The spatial filtrate is used in the spatial domain in the plane of the image by means of the direct manipulation of vecinos pixels with the help of convolution nuclei (Andrews, Hunt, 1987). In the filtrate in the frequency domain, if it neglects the presence of interference in the image and the restoration takes place the basis of the frequency recovery of the correction filter, which was configured for the inverse of the frequency response [7]. This reverse filtrate is developed in the field of frequency with the help of FFT. However, the image restoration by direct inversion was poorly planted due to the presence of observation noise [8-9]. The direct inversion caused the oscillation due to the noise amplification solution [10]. Stephen E. Reichenbach et al. has used the corresponding spatial frequency domain acquisition model. This model presents itself as convention cores for the restoration of images of Advanced Radiometer of Muy Alta Resolución (AVHRR). Small granos were carried out efficiently by convoluting them to correct the degradations and increase the apparent resolution of the image. In this restoration, the image fidelity of the convolution cores subject to explicit restrictions on the space support and the resolution of the core is maximized. It was designed with the greatest resolution that the image to perform a partial reconstruction for the geometric correction and other reassignment operations [11].

To overcome the problems of the reverse filter, use the Wiener filter. Require expands a priori information i. mi. spectra of the original image and noise. [5]. In 1977, A.K.Jain proposed that the execution of the Wiener filter become numerically blocked from a fast sinusoidal transformation algorithm that is related to the Fourier rapid transformation algorithm (FFT). The factory offers a fast Wiener restoration scheme for large size images with stretch PSF..

\section{III.SYSTEM ARCHITECTURE}

The architecture of the system of the proposed method of disclosure or basic procedure to estimate the directions of illumination of underwater images and deal with the illumination problem by standardization. Secondly, the underwater image blur was removed using the histogram equalization algorithm and, finally, mixed the two results, the restored and acquired image 


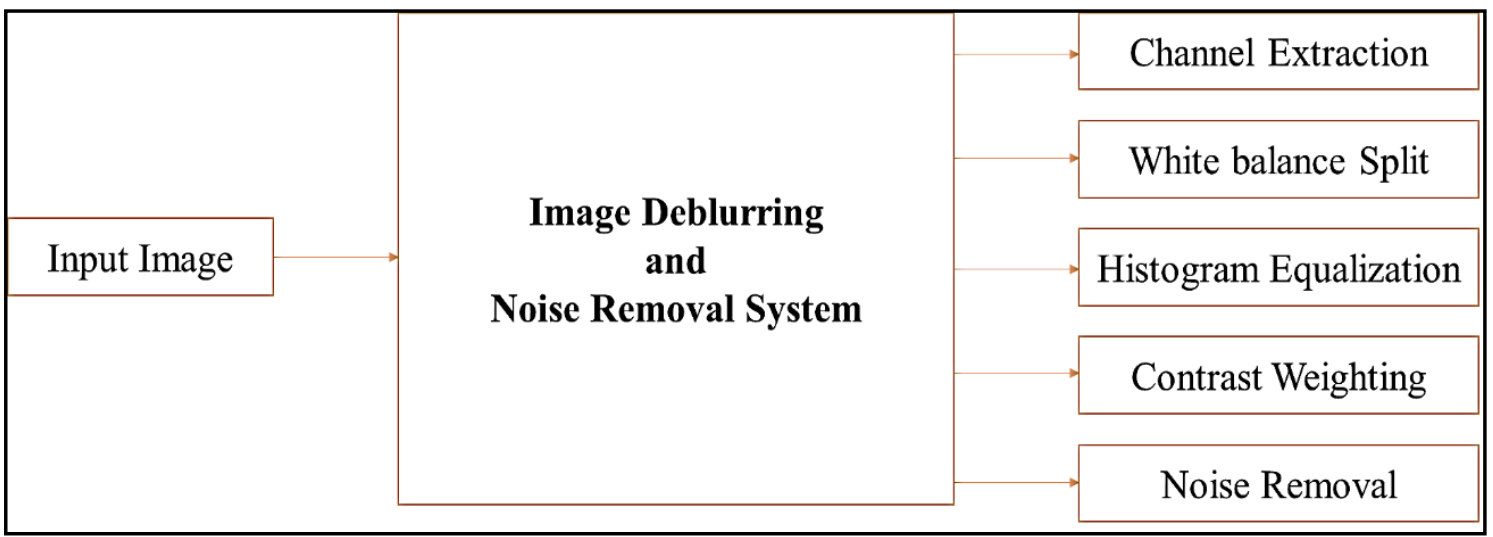

Figure 2: System Architecture

\section{IMPLEMENTATION AND DISCUSSION:}

\section{Step 1 Channel Extraction}

It is necessary to extract the different channels from the image that can be used at the end to mix the image enhanced with existing colors. Display each cor channel separately, along with the original RGB image. Notice that each plane of the body separated into a figure contains a white area. Or white corresponds to the highest values (purest shade) of each cor separately. For example, the image of red channel, or white represents a higher concentration of values of pure red. As the red mixes as green or blue, gray pixels appear. A present region of the image shows pixel values that do not contain values of red, value, $\mathrm{R}==0$

\section{Step 2 White balancing}

White color balance and the global configuration of the intensity of the cores (normally red, green and blue primary cores). An important objective of configuring and rendering specific cores, especially neutral cores, correctly. Therefore, the general method is sometimes called the balance of gray, neutral balance or balance of white. The balance of cores alters the general mixture of cores in an image and is used for correction of cores. Generalized versions of core balance are used to correct different cores on neutral days or to deliberately alter them for effect. We use the balance of cores in images in tons of gray to form several formation of different cores.

\section{Step 3 Histogram Equalization}

A histogram equalization is used to increase or contrast. It is not necessary for the contrast to always be increased here. There may be some cases in which to equalize the previous histogram. Cases, or contrast is reduced. Let's start to equalize the histogram taking the image below as a simple image.

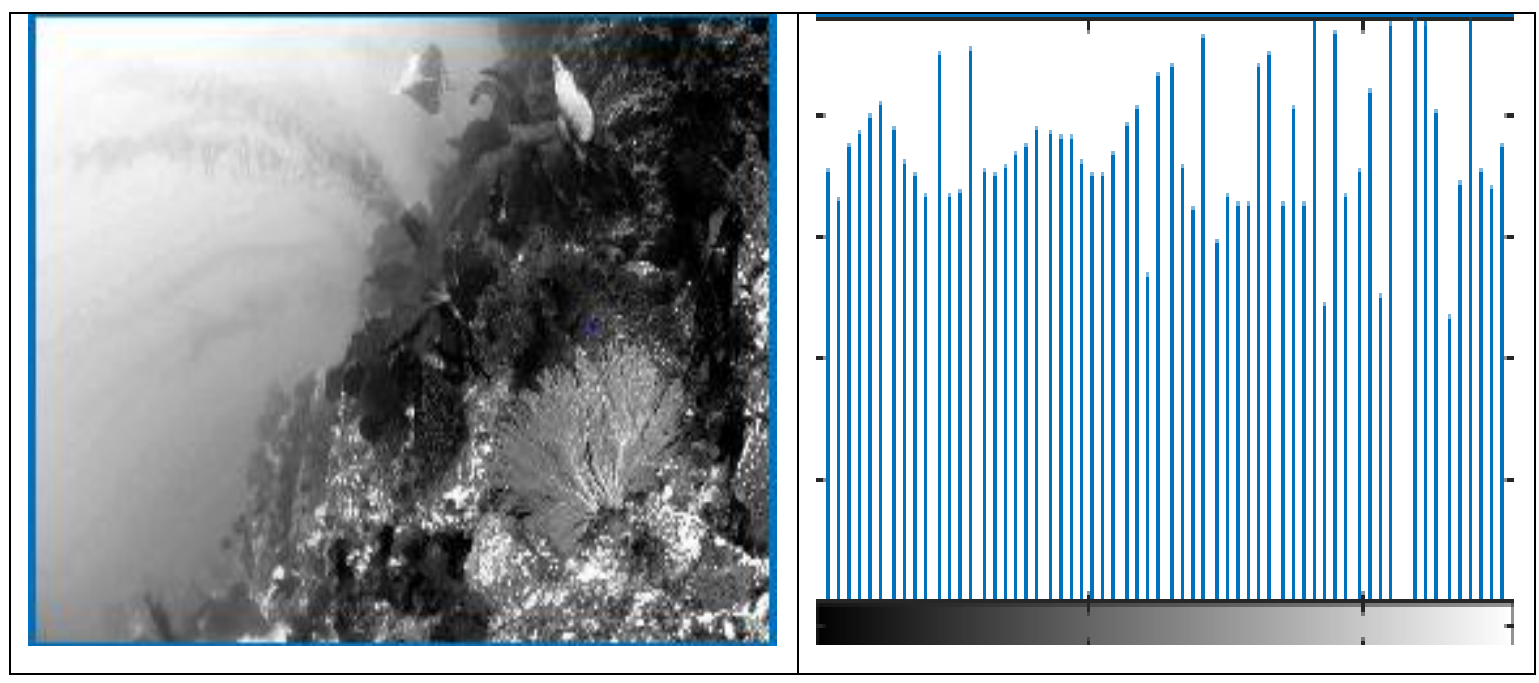


Figure 3: Histogram Equalisation

Histogram levelling is a strategy for modifying image intensities to contrast enhance. Let $f$ a given image represented to as a mr by mc matrix of whole number pixel intensities extending from 0 to $\mathrm{L}-1$. $\mathrm{L}$ is the quantity of conceivable intensity value, frequently 256 . Let p signify the standardized histogram of "f" with a receptacle for every conceivable intensities. So

The histogram equalized image $\mathrm{g}$ will be defined by

$g_{i, j}=$ floor $\left((L-1) \sum_{n=0}^{f_{i, j}} p_{n}\right) \quad p_{n}=\frac{\text { number of pixels with intensity } n}{\text { total number of pixels }} \quad n=0,1, \ldots, L-1$.

where floor() rounds down to the near by integer. This is equivalent to transforming the pixel intensities, $\mathrm{k}$, of $\mathrm{f}$ by the function

$T(k)=$ floor $\left((L-1) \sum_{n=0}^{k} p_{n}\right)$

The motivation for this transformation comes from thinking about the intensities as continuous random variables $\mathrm{X}, \mathrm{Y}$ and $[0, \mathrm{~L}-1]$ with $\mathrm{Y}$ defined by

$$
Y=T(X)=(L-1) \int_{0}^{X} p_{X}(x) d x
$$

Step 4 Fusion

The fusion of images generally involves selecting the most informative areas of the original images and combining these local areas to obtain the merged output images. A direct approach is the fusion of the input images as a weighted combination of the input images. Input images cannot be merged by calculating a weighted average across each pixel using weights calculated from quality metrics.

The merger can be used to improve the shortcomings of some existing improvement methods. There are several techniques for merging images and the selection of one in particular depends on the application. The merger problem is in reality how to define the weights and combination rules for the fusion process. A simple approach to the merger is to build a composite image as a weighted promise of the original / entry images. Weights are calculated on the basis of prominence dictated by the particular view range.

\section{ANALYSIS OF RESULT}

Above figure shows gray scale enhanced image obtained using fusion of white balance based split images. 


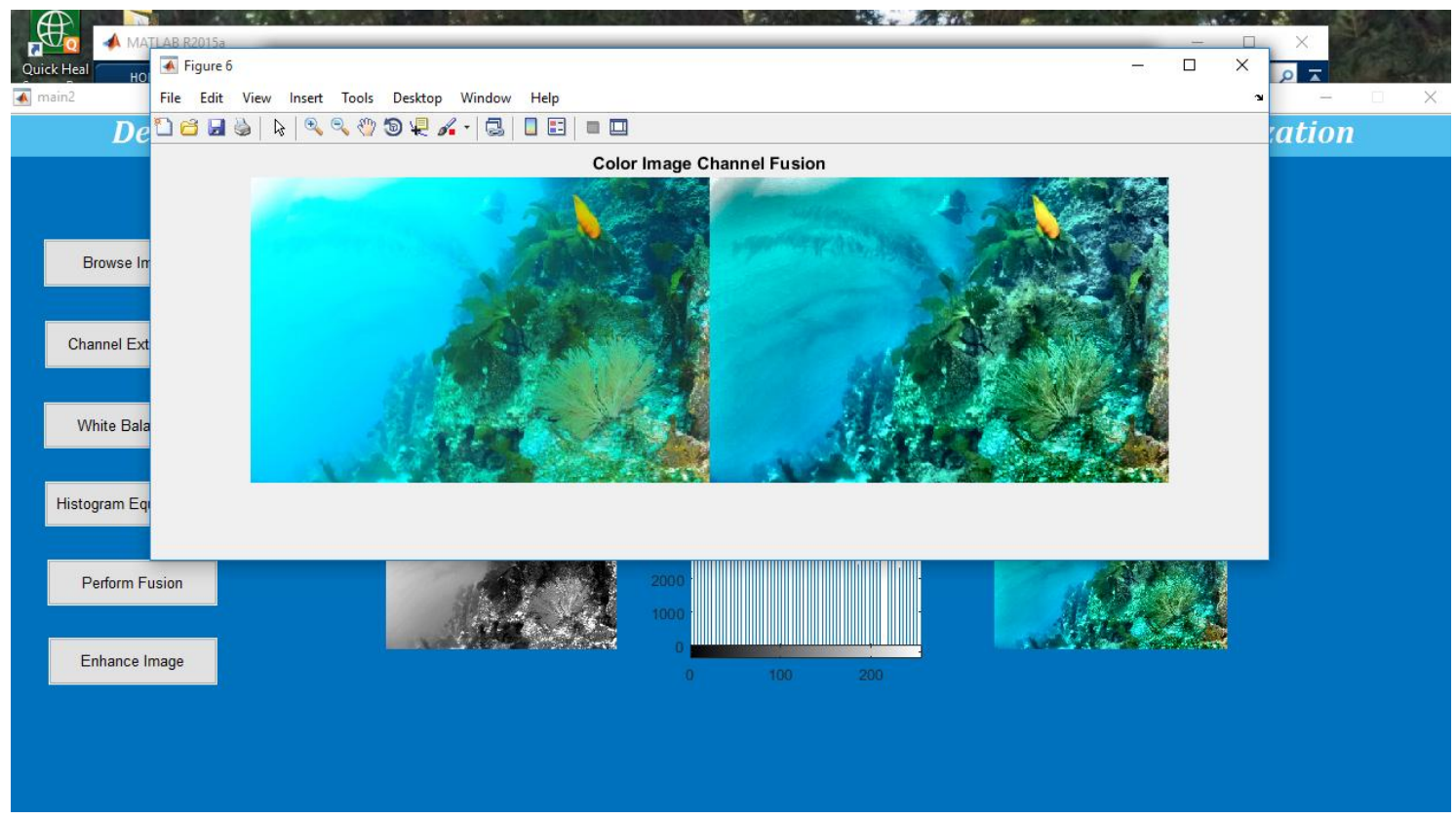

Figure 4GUI of System Architecture

Finally we get color enhance image by fusing the RGB Panels with enhanced gray scale image.

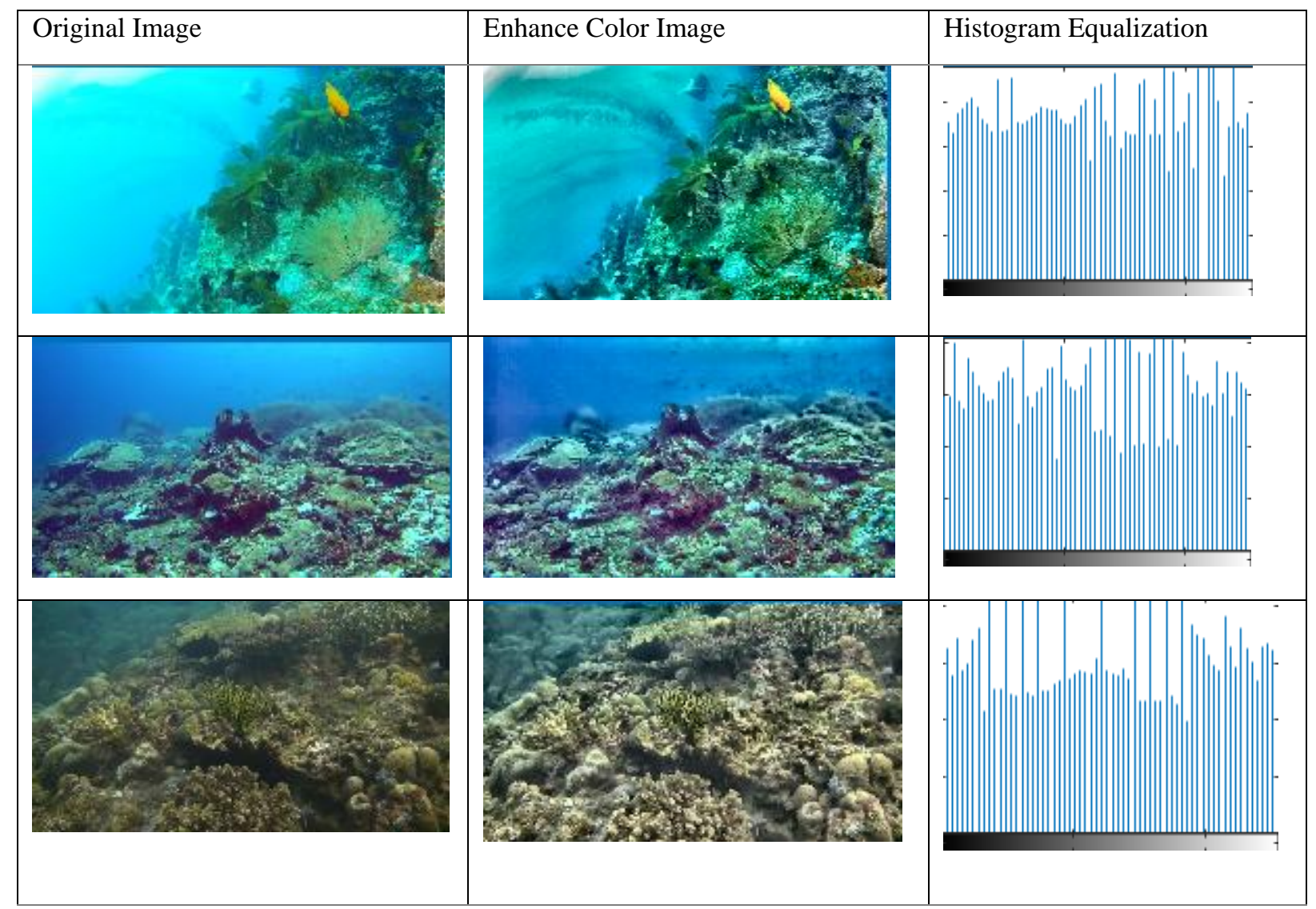

Figure 5 Result of System Architecture

\section{CONCLUSION}

We have introduced an elective way to deal with upgrade submerged videos and images. We proceed expands on the combination standard and doesn't need extra data than the single original image. We have appeared in our analyses that our methodology can improve a wide scope of submerged image (for example various cameras, profundities, light conditions) with high precision, having the option to recuperate significant blurred highlights and edges. Also, just because, we show the utility and 
significance of the proposed image enhancement method for a several challenging underwater computer vision applications. We have utilized histogram evening out as a center strategy to use contrast enhancement in images to improve the precision of picture demonstrated in above outcome examination. In view of framework results our proposed technique can re-establish 79\% of lost information happened to differentiate issues in submerged pictures. Detriment of the method Right now the framework execution speed isn't comparable to the speed of casings in video so it will require time improvement for HDR contrast upgrades.

\section{REFERENCES}

[1]. Ancuti, C., Ancuti, C. O., Haber, T., Bekaert, P. 2012. Enhancing underwater images and video by fusion. IEEE International Conference on Computer Vision and Pattern Recognition (CVPR). Providence, RI. 16-21 June 2012. pp. 81-88.

[2]. Anthoni, J. F. 2012. Water and Light in Underwater Photography. http://www.seafriends.org.nz/phgraph/water.htm, excessed on September 2012.

[3]. Arici, T., Dikbas, S., Altunbasak, Y. 2009. A Histogram Modification Framework and Its Application for Image Contrast Enhancement. IEEE Transactions on Image Processing, September 2009, 18(9), pp. 1921-1935.

[4]. Arnold-Bos, A., Malkasse, J. P., and Kerven, G. 2005a. A preprocessing framework for automatic underwater images denoising. Proceedings of the European Conference on Propagation and Systems.

[5]. Brest, France. March 2005. Arnold-Bos, A., Malkasset, J. P., Kervern, G. 2005b. Towards a model-free denoising of underwater optical images. Proceedings of the IEEE Europe Oceans Conference, Brest, France. June 2005. Vol. 1, pp. 527-532.

[6]. Bazeille, S., Quidu, I., Jaulin, L., Malkasse, J. P. 2006. Automatic underwater image preprocessing. Proceedings of the Caracterisation du Milieu Marin (CMM '06). Brest, France, 16-19 October 2006.

[7]. Foresti, G. L. 2001. Visual inspection of sea bottom structures by an autonomous underwater vehicle. IEEE Transaction on Systems, Man and Cybernetics, Part B, October 2001. 31(5), pp. 691-705.

[8]. Garg, R., Mittal, B., Garg, S. 2011. Histogram Equalization Techniques for Image Enhancement. International Journal of Electronics and Communication Technology, March 2011, 2(1), pp. 107-111.

[9]. Gasparini, F. and Schettini, R. 2003. Color Correction for Digital Photographs. IEEE 12th International Conference on Image Analysis and Processing (ICIAP 2003).

[10]. Mantova, Italy. 17-19 September 2003. pp. 646-651. Gonzalez, R. C. and Woods, R. E. 1992. Digital Image Processing, Addison-Wesley Pub (Sd), Reading, MA, March 1992, 3rd edition.

[11]. Gribbon, K. T. and Bailey, D. G. 2004. A Novel Approach to Real-time Bilinear Interpolation. Proceeding of IEEE International Conference on Field Programmable Technology. 28-30 January 2004, pp. 126-131.

[12]. Hitam, M. S., Yussof, W. N. J. W., Awalludin, E. A., Bachok, Z. 2013. Mixture Contrast Limited Adaptive Histogram Equalization for Underwater Image Enhancement. IEEE International Conference on Computer Applications Technology (ICCAT). Sousse, 20-22 January 2013, pp. 1-5.

[13]. Horváth, G. and Varjú, D. 1995. Underwater refraction-polarization patterns of skylight perceived by aquatic animals through Snell's window of the flat water surface. Journal of Vision Research, June 1995. 35(12), pp. 1651-1666.

[14]. Hsieh, C. H., Chen, B. C., Lin, C. M., Zhao, Q. 2010. Detail aware contrast enhancement with linear image fusion. IEEE International Symposium on Aware Computing (ISAC). Tainan. 1-4 November 2010. pp. 1-5. 